\title{
Evaluation of the Influence of Lightning Overvoltage Propagation on Wind Turbine and "Mix" Overhead-Cable Lines
}

\author{
Chung Thanh Pham \\ Hanoi University of Science and Technology, Hanoi, Vietnam \\ *Email: chung.phamthanh1@hust.edu.vn
}

\begin{abstract}
Due to the growing demand for new and renewable energies and a favorable geographical position, Vietnam is constructing many wind farms. Besides the advantages of being environmentally friendly and the primary endless source of materials, wind farms have disadvantages, for example, high-structured wind towers are usually built in high, empty places and the resistivity of large soil is vulnerable to lightning damage. In this research, we investigate the effects of overvoltage due to lightning strikes on the wings of wind turbines and propagation causing overvoltage on the insulation of cable in the control cable and mixed-lines. The paper also considers the overvoltage protection measures propagated into the insulation equipment and cable insulation using EMTP-RV simulation software.
\end{abstract}

Keywords: Lightning surge, EMTP- RV simulation, wind turbine, mixed line.

\section{Introduction}

Among renewable energy sources, wind energy is considered to be the most promising source because of its rich potential, easy to exploit on a large scale, environmental friendliness, and low negative impact on society. Therefore, this energy source has been and will be developed by many countries around the world, including Vietnam.

However, the use of wind turbines for power generation also has some disadvantages in terms of lightning protection [1].

Firstly, wind turbines are tall structures of up to more than 100 meters in height, wind turbines are frequently placed at locations that are highly exposed to lightning stroke.

Secondly, wind farms are often built in places with high resistivity soil, which makes it very difficult to dissipate lightning current. The lightning rod installed on the wing is always moving during the operation of the wind turbine. When lightning strikes the wind turbine, the path of lightning current through the grounding pole can cause dangerous induced lightning overvoltage to the internal components of the wind turbine [2].

Finally, wind turbines are connected to each other to form a wind farm that supplies power to the system grid (or supplies power to local loads) via overhead medium voltage lines. Therefore, when lightning strikes any wind turbine in wind farms or hits an overhead medium voltage line connecting the wind farm to the grid, there may be overvoltage due to dangerous lightning propagation in the wind farm power grid.

According to IEC standard TR61400-24 [3], more than $50 \%$ of failures are due to direct, overvoltage induced and propagating lightning strikes in wind turbine equipment occur in low voltage, controlled circuits and signal transmission circuits. These failures cause serious problems, severe economic losses, and significantly affecting system reliability.

Therefore, the paper studies the effect of overvoltage caused by direct lightning strokes on the blades of wind turbines and propagating large overvoltage on the insulation of low voltage control devices, i.e. $22 \mathrm{kV}$ cables connected to the medium voltage grid of the power system (mixed-lines structure between cables and overhead lines (OHL)) as shown in Fig. 1. EMTP-RV was used to calculate and evaluate voltage transients caused by lightning strikes transmitted to the wind turbine. At the same time, the effectiveness of the use of overvoltage protection devices propagated to the control equipment and the basic insulation of the cable and the insulation of the cable sheath are taken into account.

\section{Wind Turbine Description}

The generator of the $2 \mathrm{MW}$ wind turbine has a rated voltage of $0.69 \mathrm{kV}$ with a frequency of $50 \mathrm{~Hz}$ as shown in Fig. 2. The blade length is $63 \mathrm{~m}$ and is placed on a $135 \mathrm{~m}$ high cylinder as shown in Fig. 9.

ISSN: 2734-9373

https://doi.org/10.51316/jst.152.ssad.2021.31.2.14

Received: July 15, 2020; accepted: August 13, 2021 


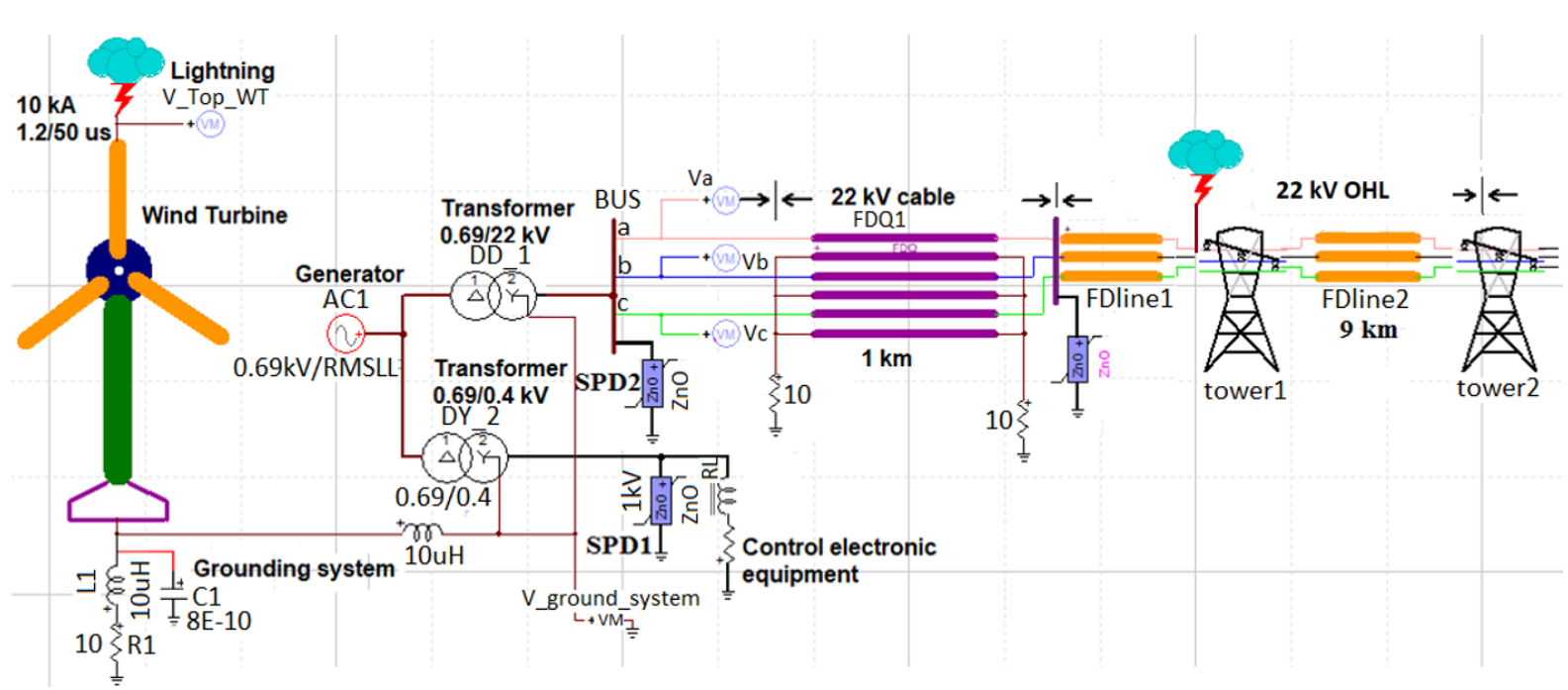

Fig. 1. EMTP- RV simulation model

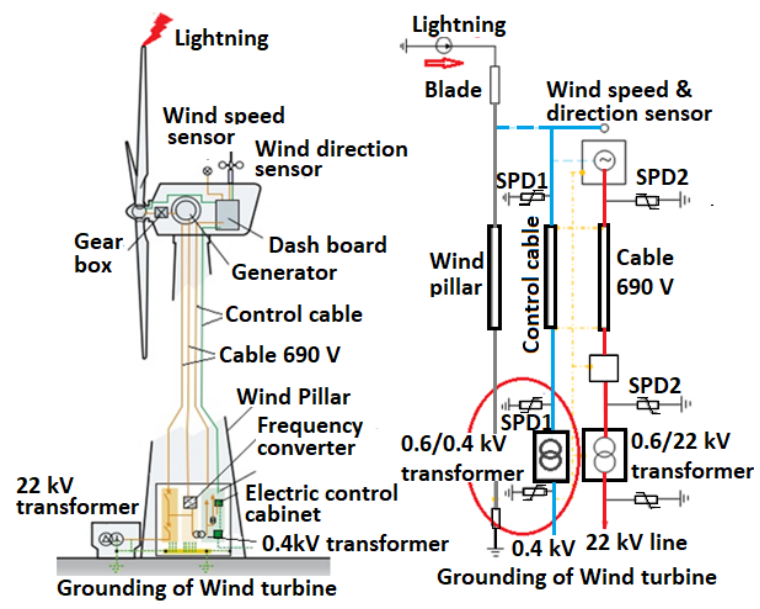

Fig. 2. Layout diagram of elements and lightning protection system of the wind turbine.

The grounding system of the wind turbine is simulated with an average ground electrode resistivity value $(\rho=100 \Omega \mathrm{m})$ and a ground resistance value of $10 \Omega$, according to model 1 [4].

In this research, we model the lightning current with an amplitude $10 \mathrm{kA}$, slope of $1.2 / 50 \mu \mathrm{s}$. According to statistics, about $80 \%$ of direct lightning strikes are $10 \mathrm{kA}$ lightning current [5].

\section{EMTP- RV Model}

The EMTP program has been used to study electromagnetic transients at the scale of large power systems or in any electrical network.

The following briefly explains the most important models used in this paper.

\subsection{The Generator in Wind Turbine}

$v(t)=V_{m} \cos (\omega t+\theta), \omega=2 \pi f, \theta=$ Phase

\begin{tabular}{|c|c|c|c|}
\hline Phase A & Phase B & Phase C & \\
\hline$V _ { m } \longdiv { 0 . 6 9 }$ & 0.69 & 0.69 & $\sqrt{\text { KVRMSLL } v}$ \\
\hline $\mathrm{f} 50$ & 50 & 50 & $\Delta D$ Hertz \\
\hline$\theta \longdiv { 0 }$ & -120 & 120 & $\longdiv { \operatorname { d e g } v }$ \\
\hline$t_{\text {star }}^{-1}$ & -1 & -1 & $s v$ \\
\hline$t_{\text {stop }} 1$ 1E15 & $1 E 15$ & 1E15 & $s v$ \\
\hline \multicolumn{4}{|l|}{$\nabla$ balanced } \\
\hline Start in steady-state & Never stop & & \\
\hline
\end{tabular}

Fig. 3. The generator declared in EMTP- RV

Using a synchronous generator with a capacity of $2 \mathrm{MW}$, rated voltage $690 \mathrm{~V}$, frequency $50 \mathrm{~Hz}$ declared in EMTP-RV in Fig. 3. Power supplied for 2 transformers $0.69 \mathrm{kV} / 22 \mathrm{kV}$ and $0.69 \mathrm{kV} / 0.4 \mathrm{kV}$.

\subsection{Transformers}

A $690 \mathrm{~V} \mathrm{/} 22 \mathrm{kV}$ boost transformer $(\Delta-\mathrm{Y}$ connection) is placed inside the wind turbine or installed rather close to the turbine. The generator terminal voltage is increased from $690 \mathrm{~V}$ to $22 \mathrm{kV}$ connected to a $1 \mathrm{~km}$ long underground cable and connected to the medium-voltage line. The terminals of the cable sheath are connected to the grounding system of the wind turbine.

The 0.25 MVA transformer ( $\Delta-\mathrm{Y}$ connection) changes the generator terminal voltage from $690 \mathrm{~V}$ to $0.4 \mathrm{kV}$ to supply control devices (RL). Fig. 4 is a datasheet of 2 transformers in the EMTP-RV. 
JST: Smart Systems and Devices

Volume 31, Issue 2, September 2021, 108-116

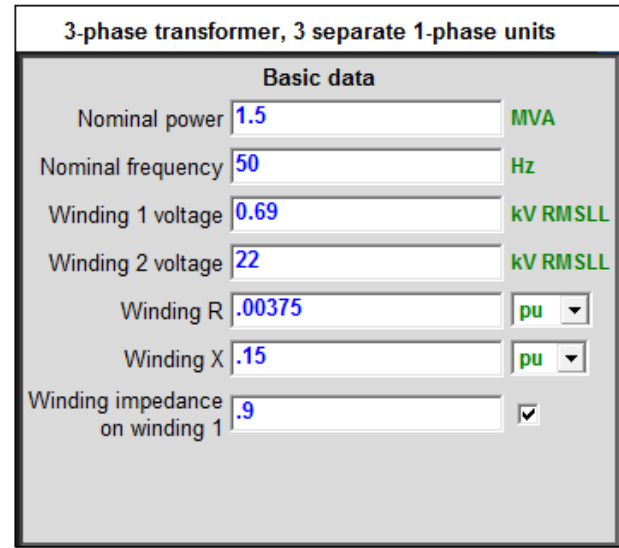

(a)

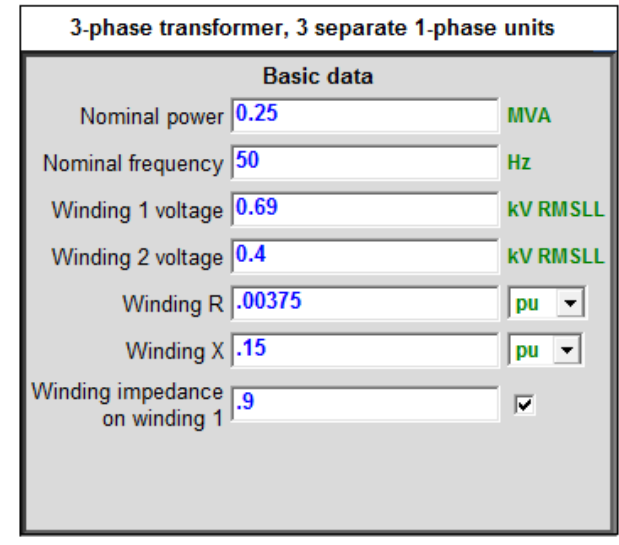

(b)

Fig. 4 (a) Transformer $0.69 \mathrm{kV} / 22 \mathrm{kV}$, (b) Transformer $0.69 \mathrm{kV} / 0.4 \mathrm{kV}$.

\subsection{Cables}

There are 2 types of cables: $0.4 \mathrm{kV}$ control cable (RG58A/U-0.48 $\mathrm{mm}^{2}$ ) and $22 \mathrm{kV}$ cable (Cu-XLPE$860 \mathrm{~mm}^{2}$ ). Single cable line CL uses 3 separate cores, $860 \mathrm{~mm}^{2} \mathrm{Cu}, 22 \mathrm{kV}$ insulation of XLPE cables arranged horizontally $0.25 \mathrm{~m}$ apart and $1.1 \mathrm{~m}$ deep in the ground $1 \mathrm{~km}$ long. Table 1 is the geometrical, physical, structure and declared data of the underground cable line in the EMTP-RV simulation shown in Fig. 5 and Fig. 6.

Table 1. Parameters of Cu-XLPE- $860 \mathrm{~mm}^{2}$ cable

\begin{tabular}{|c|c|c|c|}
\hline $\mathrm{r}_{2}$ & $12.54 \mathrm{~mm}$ & $\varepsilon_{\mathrm{r} 1}$ & 3.5 \\
\hline $\mathrm{r}_{3}$ & $22.735 \mathrm{~mm}$ & $\varepsilon_{\mathrm{r} 2}$ & 2.0 \\
\hline $\mathrm{r}_{4}$ & $26.225 \mathrm{~mm}$ & $\rho_{\mathrm{c}}$ & $1,7.10^{-8} \Omega \mathrm{m}$ \\
\hline $\mathrm{r}_{5}$ & $29.335 \mathrm{~mm}$ & $\rho_{\mathrm{s}}$ & $2,1.10^{-7} \Omega \mathrm{m}$ \\
\hline
\end{tabular}

\subsection{Lightning Current Source}

Lightning stroke is modeled using the current

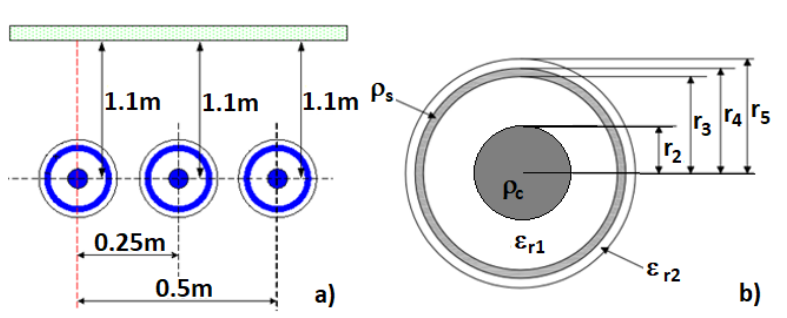

Fig. 5 (a) The layout of underground cable; (b) Structure of the underground cable.

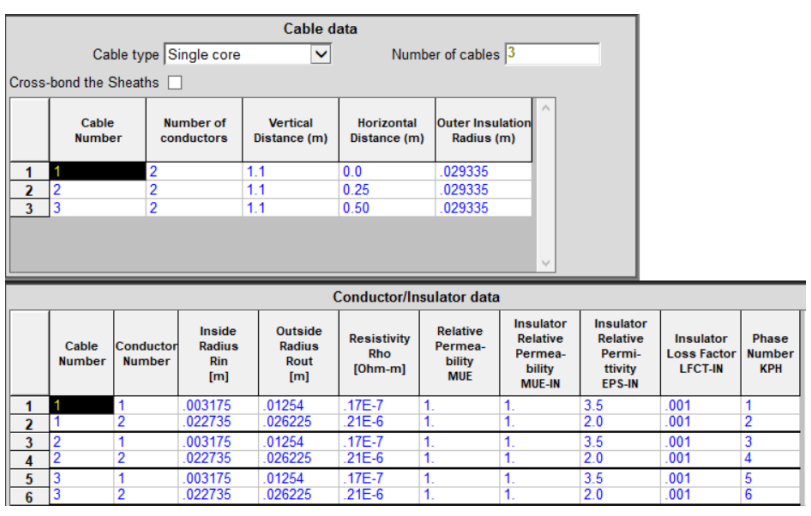

Fig. 6. Data table of underground cable in EMTP- RV.

source in parallel with the resistance that represents the impedance of the lightning channel. Resistance of 400 $\Omega$ has been widely accepted to be a suitable estimate for the impedance of lightning channel [6].

Representative lightning current wave-shapes i.e. ramped, double exponential, and concave are alternatingly used in literature. However, they tend to produce overvoltage with significantly different peak values and time to crest [7].

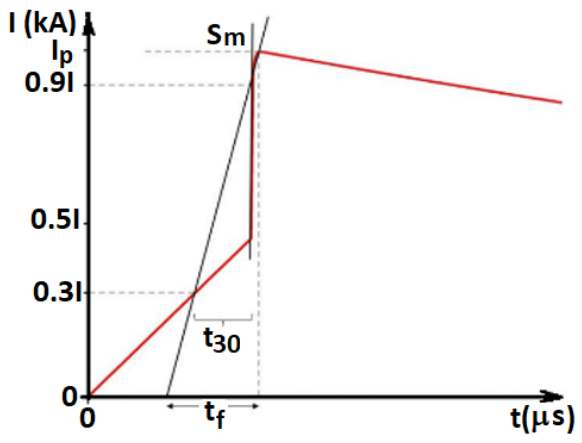

Fig. 7. Concave front lightning wave-shape recommended by CIGRE for analysis of lightning transients [8]. 
An upwardly concave lightning current waveform, shown in Fig. 7, is closest to the actual recorded lightning current wave-shapes given in [8] and [9]. It can be mathematically represented by the formula (1),

$$
i(t)= \begin{cases}A t+B t^{n}, & t<t_{n} \\ I_{1} e^{-\frac{\left(t-t_{n}\right)}{t_{1}}}-I_{2} e^{-\frac{\left(t-t_{n}\right)}{t_{2}}}, t>t_{n}\end{cases}
$$

The wave shape constants $A, B, t_{n}, n, I_{1}, I_{2}, t_{l}$ and $t_{2}$ are listed in Table 2 and are derived as per the procedure described in [8].

The probability of occurrence of lightning current exceeding $10 \mathrm{kA}$ is $80 \%$ [5]. Therefore, $10 \mathrm{kA}$ is chosen as the peak intensity of the lightning strike on the top of the wind turbine propeller suggested by the IEEE modeling guide [10].

The CIGRE pulse source is used to simulate lightning current with amplitude of $10 \mathrm{kA}$, slope of $1.2 / 50 \mu \mathrm{s}$ with the largest wave front slope varying with the maximum lightning current [11] which is calculated by the formula (2) and is shown in Fig. 8 with the case of lightning hitting the top of the wind turbine blade,

$$
S_{m}=1.4 I^{0.77}
$$

where $S_{m}$ is the maximum rate of rise $[\mathrm{kA} / \mu \mathrm{s}], I$ is the current amplitude [kA].

Table 2. Parameters of lightning current impulse.

\begin{tabular}{|c|c|c|}
\hline \multicolumn{3}{|c|}{ Parameters of Strike } \\
\hline $\mathrm{I}_{\mathrm{p}}$ & Peak current & $10 \mathrm{kA}$ \\
\hline $\mathrm{t}_{30}$ & Front $(30 \%-90 \%)$ & $8 \mu \mathrm{s}$ \\
\hline $\mathrm{t}_{\mathrm{f}}$ & Effective front time & $1,2 \mu \mathrm{s}$ \\
\hline $\mathrm{t}_{\mathrm{h}}$ & Time to half value & $50 \mu \mathrm{s}$ \\
\hline $\mathrm{S}_{\mathrm{m}}$ & Maximum steepness & $9,1 \mathrm{kA} / \mu \mathrm{s}$ \\
\hline $\mathrm{n}$ & Wave - shape constant & 5 \\
\hline $\mathrm{A}$ & Wave - shape constant & $4,58 \mathrm{kA} / \mu \mathrm{s}$ \\
\hline $\mathrm{B}$ & Wave - shape constant & $0,1 \mathrm{kA} / \mu \mathrm{s}$ \\
\hline $\mathrm{t}_{\mathrm{n}}$ & Wave - shape constant & $1,65 \mu \mathrm{s}$ \\
\hline $\mathrm{t}_{1}$ & Wave - shape constant & $70 \mu \mathrm{s}$ \\
\hline $\mathrm{t}_{2}$ & Wave - shape constant & $0.55 \mu \mathrm{s}$ \\
\hline $\mathrm{I}_{1}$ & Wave - shape constant & $10,11 \mathrm{kA}$ \\
\hline $\mathrm{I}_{2}$ & Wave - shape constant & $5,11 \mathrm{kA}$ \\
\hline
\end{tabular}

\subsection{The Tubular Steelturbine}

The tubular steel turbine, of $15 \mathrm{~mm}$ thick and $135 \mathrm{~m}$ high is simulated in EMTP-RV as shown in Fig. 9 with the wave impedance is $200 \Omega$ and a velocity of propagation of $3.10^{8} \mathrm{~m} / \mathrm{s}$ connected to a 63 meters long blade of the wind turbine.

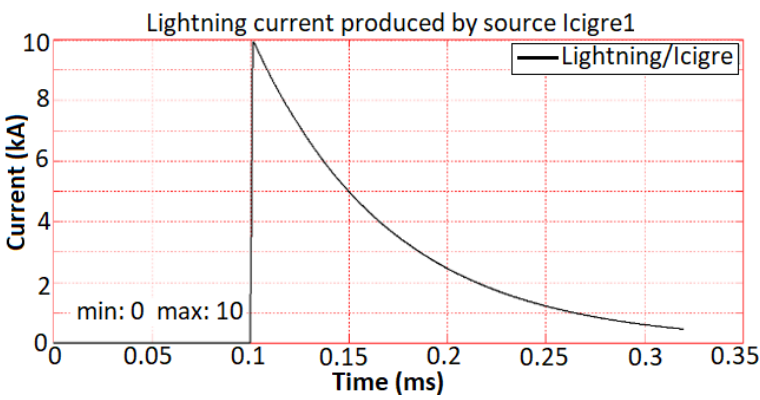

Fig. 8. Lightning current produced by source CIGRE
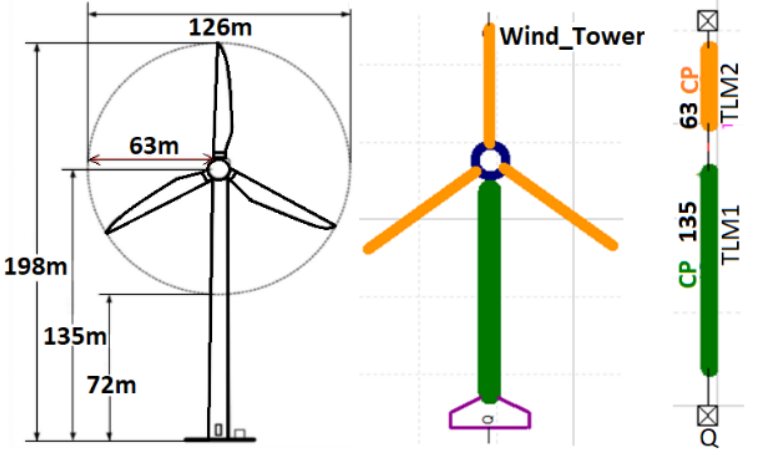

Fig. 9. Dimensions of the wind turbine

\subsection{Surge protective device modeling}

For the purpose of protecting the overvoltage propagated from the grounding system of the wind turbine through the neutral point of the transformer $0.69 \mathrm{kV} / 0.4 \mathrm{kV}$ through the control cable to supply power to the control devices

The system uses the SPD1- Surge Protective Device modeling of an LPZ0A- LPZ1, which is made in Japan arrester for a $0.4 \mathrm{kV}$ network MCOV $1 \mathrm{kV}$ $(d=1.8 \mathrm{~m}$ and $n=1)$ use $\mathrm{ZnO}$ arrester model based on IEEE surge protective device working group [12]. The parameters of the SPD1 simulation circuit are calculated according to the formula (3) and is shown in Fig. 10 with the V-A characteristics as shown in Table 3 .

$$
\begin{aligned}
& L_{1}(\mu H)=15 \frac{d}{n} ; R_{1}()=65 \frac{d}{n} \\
& L_{0}(\mu H)=0,2 \frac{d}{n} ; R_{0}()=100 \frac{d}{n} \\
& C_{0}(p F)=100 \frac{n}{d}
\end{aligned}
$$


where $d(\mathrm{~m})$ is the height of the surge arrester, $n$ number of parallel columns.

Table 3. V-A characteristics of SPD1 and SPD2.

\begin{tabular}{|c|c|c|}
\hline Current (A) & Volts Peak- SPD & $\begin{array}{c}\text { Volts Peak- } \\
\text { SVL }\end{array}$ \\
\hline 0.00001 & 238.1 & 2428.6 \\
\hline 0.00003 & 1190.5 & 12142.9 \\
\hline 0.0001 & 1666.7 & 17000.0 \\
\hline 0.0005 & 1845.2 & 18821.4 \\
\hline 0.01 & 1904.8 & 19428.6 \\
\hline 1 & 2000.0 & 20400.0 \\
\hline 10 & 2113.1 & 21553.6 \\
\hline 125 & 2345.2 & 23921.4 \\
\hline 250 & 2416.7 & 24650.0 \\
\hline 500 & 2500.0 & 25500.0 \\
\hline 1000 & 2642.9 & 26957.1 \\
\hline 1500 & 2738.1 & 27928.6 \\
\hline 3000 & 2881.0 & 29385.7 \\
\hline 5000 & 3023.8 & 30842.9 \\
\hline 10000 & 3238.1 & 33028.6 \\
\hline 20000 & 3559.5 & 36307.1 \\
\hline 40000 & 4000.0 & 40800.0 \\
\hline 100000 & 5000.0 & 51000.0 \\
\hline & & \\
\hline
\end{tabular}

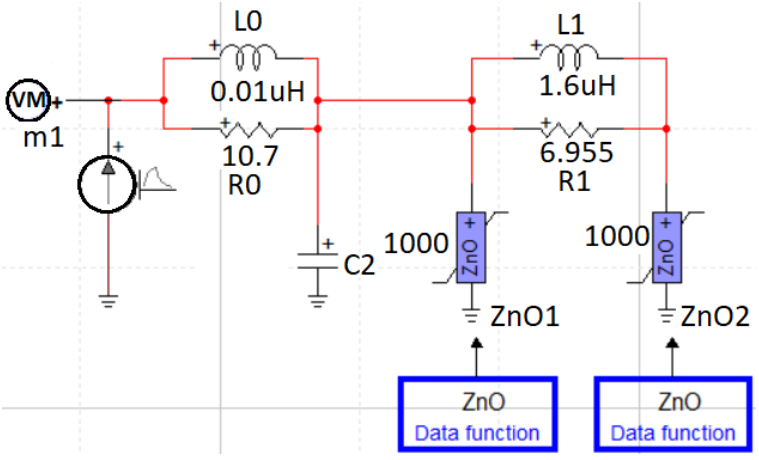

Fig. 10. SPD1 simulation model in RV.

For overvoltage protection of $22 \mathrm{kV}$ cable and sheath insulation, SPD2-24 kV MCOV $19.2 \mathrm{kV}$ is used with V-A characteristic (Table 3), Fig. 11.

\subsection{Overhead Line}

The single circuit $22 \mathrm{kV}-50 \mathrm{~Hz}$ OHL, on lattice suspension towers, with horizontally arranged phase conductors, "I" insulator strings, without shield wires and line spans up to $150 \mathrm{~m}$ (Fig. 12), is a typical European design [13]. The overall simulated tower height is $20.5 \mathrm{~m}$; phase to phase horizontal distance is $1 \mathrm{~m}$ with $20 \mathrm{~m}$ minimum ground clearance. Each phase is equipped with a AS-70/11 conductors $(\varnothing=11,4 \mathrm{~mm})$, Outer phase "I" strings are made of 3 standard $\pi-4,5$ insulators; the inner one has an additional insulator. However, arc control devices dictate the minimum gap to the horizontal truss, which is $0.51 \mathrm{~m}$.

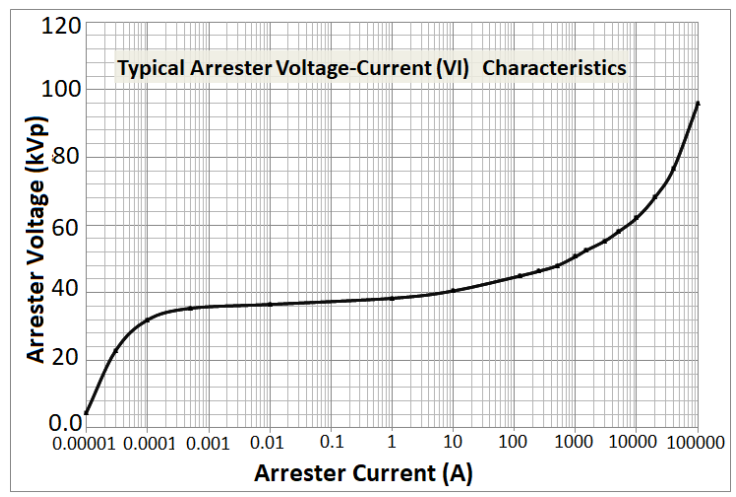

Fig. 11. V-A characteristics of SPD2.

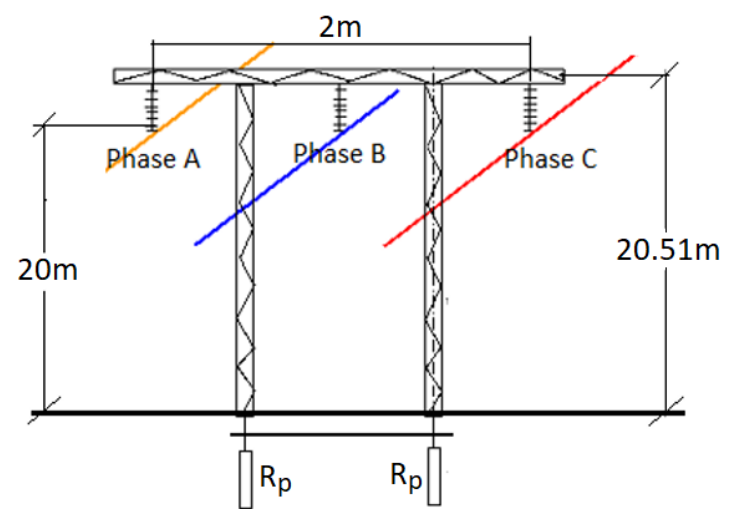

Fig. 12. Outline of the simulated $22 \mathrm{kV}$ OHL tower (not to scale).

The tower grounding system, shown in Fig. 12, is devised by an important European TSO for use in medium resistivity soils $(\rho=100 \Omega \mathrm{m})$. Model of a tower is simulated in EMTP-RV as shown in Fig. 13.

The $9 \mathrm{~km}$ of OHL adjoining the cable has been simulated in EMTP-RV as 60 line spans, each $150 \mathrm{~m}$ long, by means of the "JMARTI" frequencydependent model [14], [15]. The models of OHL spans have been obtained around the frequency of $100 \mathrm{kHz}$ for over high voltage simulation, with $100 \Omega \mathrm{m}$ earth resistivity. At the opposite end from the cable, the OHL model is "connected" to the line surge impedances; phase conductors are then terminated on a symmetrical three-phase $22 \mathrm{kV}-50 \mathrm{~Hz}$ voltage system, while without shield wires.

The physical connection between OHL and CL (downleads) is simulated by means of short, uncoupled TLs. OHL insulation breakdown has been simulated with EMTP- RV by means of the "Air gap" programming and simulation language. Line corona has been disregarded. One of the most suitable methods for the evaluation of the moment and level of arcing of spark gaps after a lightning strike is the 
integral method. The insulation is modeled by formula (4):

$$
\int_{t_{0}}^{t}\left[\left|v_{\text {gap }}(t)-V_{0}\right|\right]^{K} d t \geq D
$$

Flashover occurs when the above integral becomes greater or equal to $D$. $t_{0}$ is the time-point from which $v_{\text {gap }}$ became greater than $V_{0}$. When the voltage $v_{\text {gap }}$ goes below $V_{0}$ the integral is reset. The gap is an ideal open switch before flashover and becomes an ideal closed switch after flashover. The gap stays closed after flashover until the control signal $C$ becomes greater than 0 , in which case it will reset (open) the gap [16].

In this simulation, the spacing of the gaps is $0.28 \mathrm{~m}, \mathrm{~K}$ is $0.92, \mathrm{~V}_{0}$ is $67 \mathrm{kV}$ and $\mathrm{D}$ is $3,7.10^{-2}$.

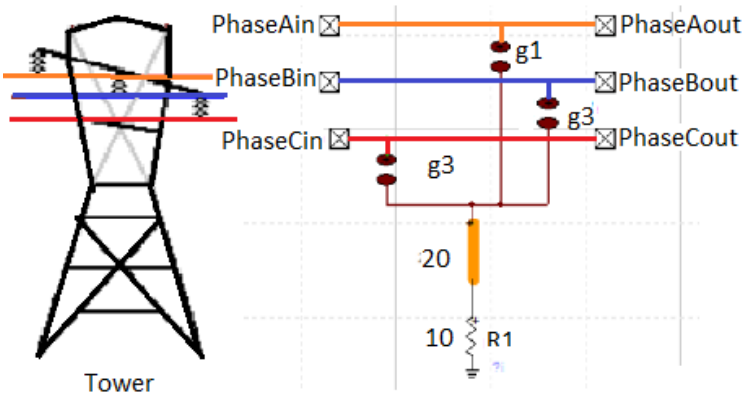

Fig. 13. Model of a tower in EMTP- RV.

\subsection{Ground Electrode}

In the low-frequency case, static analysis is usually applied, which leads to the well-known formulas for the grounding resistance $R$ [see Fig. 14a].

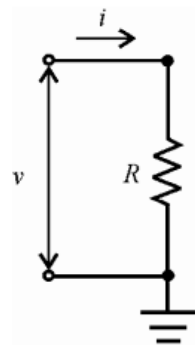

(a)

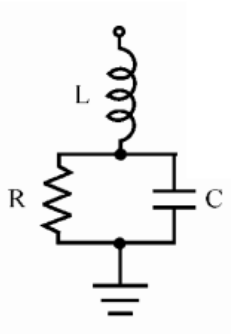

(b)
Fig. 14. Low-current models of a grounding electrode. (a) Low-frequency grounding resistance. (b) Highfrequency lumped RLC circuit.

Therefore, for the vertical electrode, according to Dwight [17] we have

$$
R=\frac{\rho}{2 \pi l}\left[\ln \left(\frac{4 l}{a}\right)-1\right]()
$$

and for the horizontal electrode, according to Sunde [4]:

$$
R=\frac{\rho}{\pi l}\left[\ln \left(\frac{2 l}{\sqrt{2 a d}}\right)-1\right]()
$$

where $\rho=100 \Omega . \mathrm{m}$ is resistivity of the earth,

$l=40 \mathrm{~m}$ is grounding electrode length,

$a=4 \cdot 10^{-3} \mathrm{~m}$ is radius,

$d=1 \mathrm{~m}$ is the depth of burial of horizontal electrode, assuming $l \gg>a$ and $l \gg d$.

A simple lumped-circuit high-frequency model suggested by Rudenberg [18] is illustrated in Fig. 14b. $R$ is computed by (7) and the grounding capacitance $C$ is computed based on the relationship [2]

$$
C(F)=\frac{\rho \varepsilon}{R}
$$

where $\varepsilon=4.10^{-11} \mathrm{~F} / \mathrm{m}$ is the permittivity of the quartz soil.

As mentioned in [4, p. 257], the inductance $L$ of a horizontal wire reduces slightly as the depth of the wire is increased. Therefore, for horizontal "wires at ordinary depths, the inductances are substantially the same as for wires at the surface" $[4, \mathrm{p} .114]$ given as:

$$
L(H)=\frac{\mu l}{2 \pi}\left[\ln \left(\frac{2 l}{a}\right)-1\right]
$$

where $\mu=1.26 .10-6 \mathrm{H} / \mathrm{m}$ is the permeability of the soil, which is usually assumed to be equal to the permeability of a vacuum. The same formula is used also for vertical electrodes [19]. The use of alternative approximation formulas for $\mathrm{L}$ is discussed in [20]. The grounding system parameters can be calculated with the grounding resistance $\left(R_{l}=10 \Omega\right)$; the inductance $\left(L_{I}=1 \cdot 10^{-4} \mathrm{H}\right)$ and the capacitance $\left(C_{I}=8 \cdot 10^{-10} \mathrm{~F}\right)$.

\section{Simulation Results}

\subsection{Case of No Protective Device}

When there is a lightning current of $10 \mathrm{kA}$, slope of $1.2 / 50 \mu$ s hits the blade of the wind turbine with the lightning current entering the tubular steel turbine and part of the lightning current dissipating into the grounding system of the pole. Part of the current through the $0.69 \mathrm{kV} / 0.4 \mathrm{kV}$ transformer neutral point ground wire causes overvoltage to propagate into the control cable.

The overvoltage at the lightning strike point on the wing of the wind turbine reaches a peak value of about 1.27 MV as shown in Fig. 15.

Fig. 16 depicts the overvoltage on the grounding system of a wind turbine. The peak overvoltage value is $466 \mathrm{kV}$ due to the high resistance grounding system.

Fig. 17 is the output voltage of the synchronous generator. We see that the spreading overvoltage does not affect the generator. 
Fig. 18 is the overvoltage on the electronic control device after the $0.69 \mathrm{kV} / 0.4 \mathrm{kV}$ transformer. Overvoltage caused by lightning with a maximum value of $3.2 \mathrm{kV}$ exceeds the threshold that the insulation of the electronic control devices can withstand.

Fig. 19 is the overvoltage across the cable sheath insulation at the input and output positions of the cable when a lightning current of $10 \mathrm{kA}, 1.2 / 50 \mu \mathrm{s}$ hits phase A of the OHL (Fig. 1). Overvoltage on cable sheath

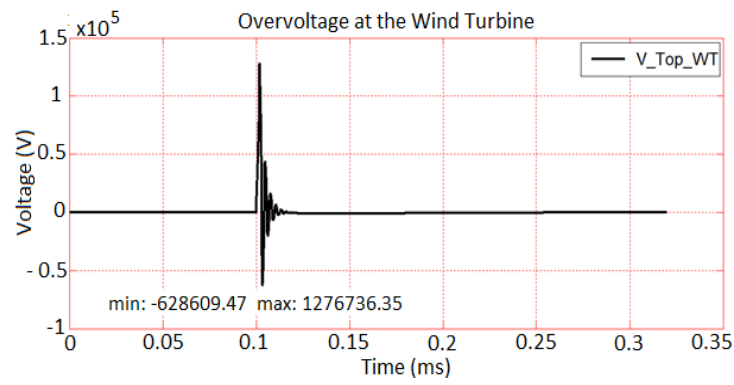

Fig. 15. Overvoltage at the wind turbine.

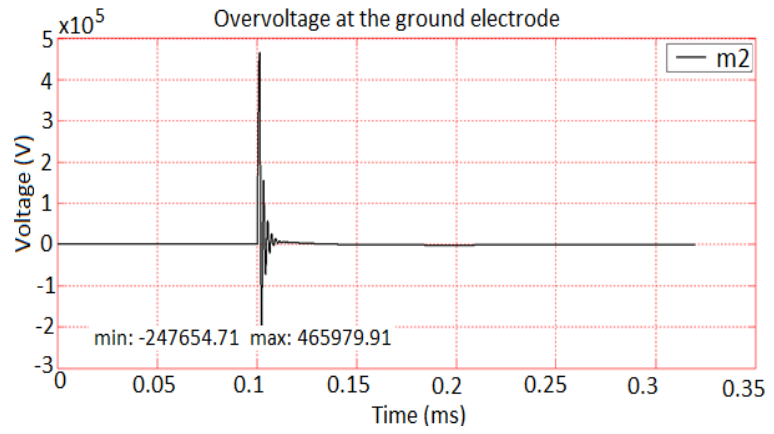

Fig. 16. Ovevoltage on the grounding system.

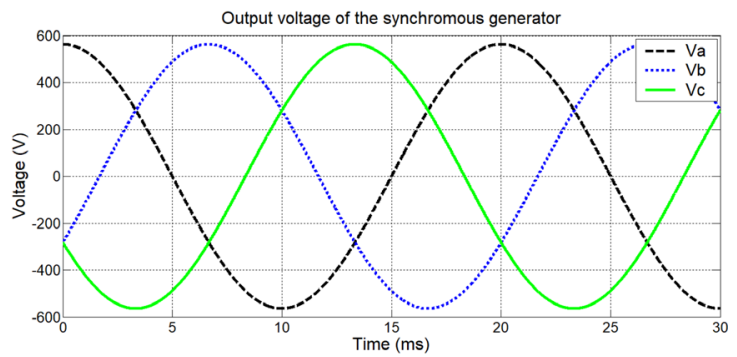

Fig. 17. Voltage at generator output.

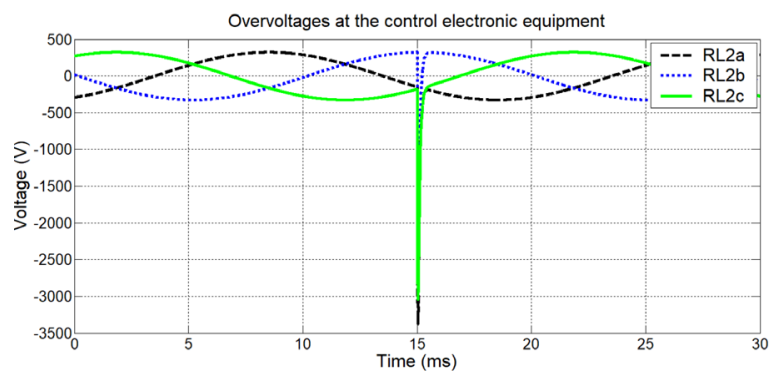

Fig. 18. Overvoltage on control devices. maximum and equal to $45 \mathrm{kV}$ exceeds the tolerable insulation level of $30 \mathrm{kV}$ for $22 \mathrm{kV}$ cable according to BIL (Basic Impulse Level) [21].

Fig. 20 is the overvoltage on the cable core at the 22 $\mathrm{kV}$ cable input location. Overvoltage with a maximum value of $254 \mathrm{kV}$ at phase $\mathrm{A}$ exceeds the standard insulation threshold of $22 \mathrm{kV}$ cables that can withstand up to $140 \mathrm{kV}$ LIWL (Lightning Impulse Withstand Level) [22].

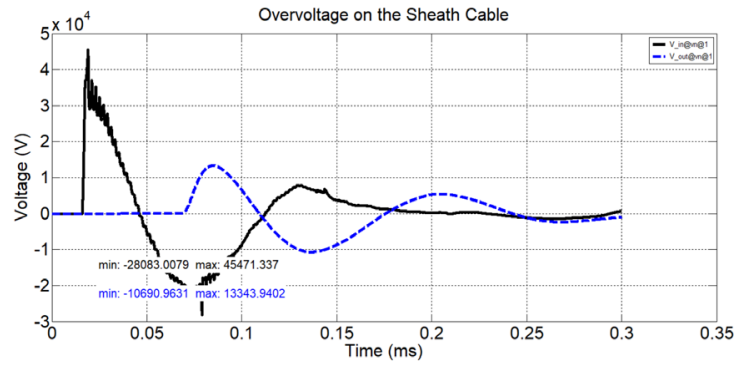

Fig. 19. Overvoltage on cable sheath insulation.

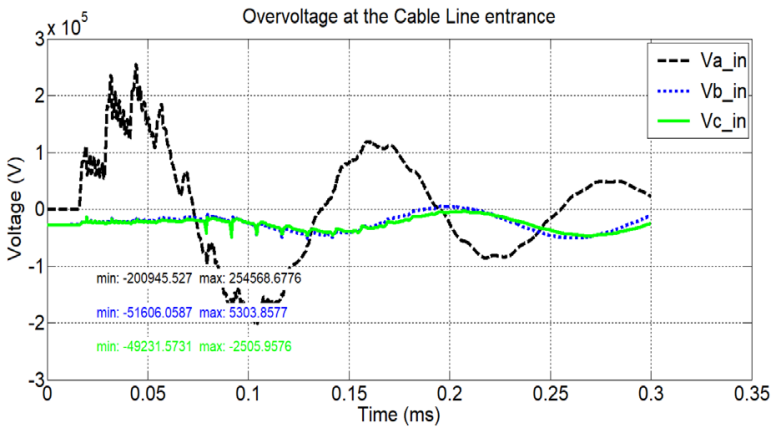

Fig. 20. Overvoltage on cable core.

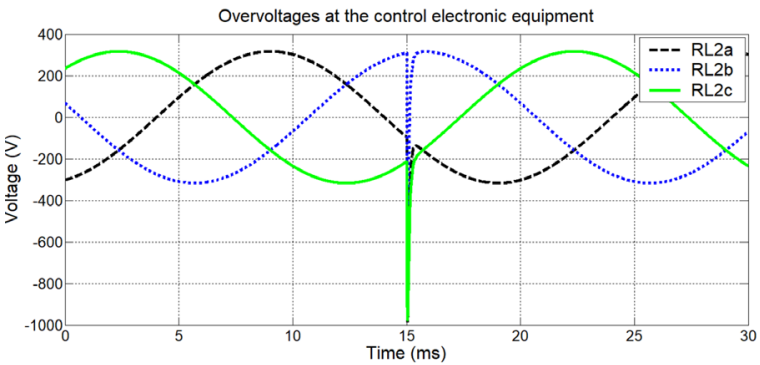

Fig. 21. Overvoltage on electronic control devices after using SPD1.

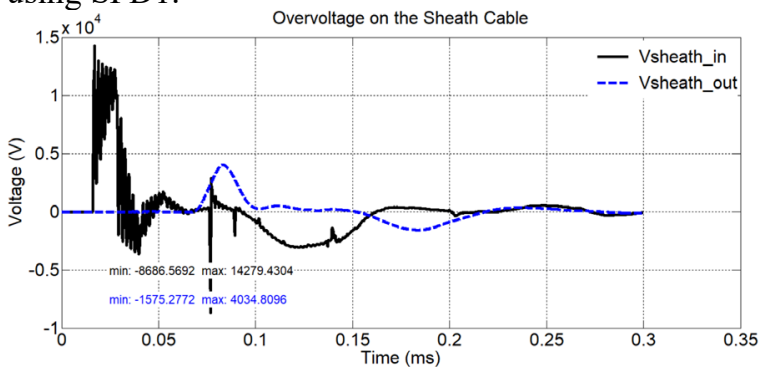

Fig. 22. Overvoltage on cable sheath after using SPD2. 


\subsection{Case of Protective Device}

In this case, the insulation of the control devices on the low voltage side and the main insulation and insulation of the cable sheath are in danger due to overvoltage propagation. For protection of electronic control equipment and of $22 \mathrm{kV}$ cables, surge arresters are used. Use SPD1 to protect electronic control devices and use SPD2 to protect cable insulation as shown in Fig. 1.

After using SPD1, the overvoltage on the electronic control devices drops to about $1 \mathrm{kV}$ which is the threshold that the insulation of the low voltage electronics can withstand as shown in Fig. 21.

After using SPD2, the overvoltage across the cable sheath insulation drops to about $14 \mathrm{kV}$ which is the threshold that the sheath insulation can withstand as shown in Fig. 22.

Overvoltage on the core of the cable is reduced to $55 \mathrm{kV}$, the main insulation of the cable core ensures safety as shown in Fig. 23.

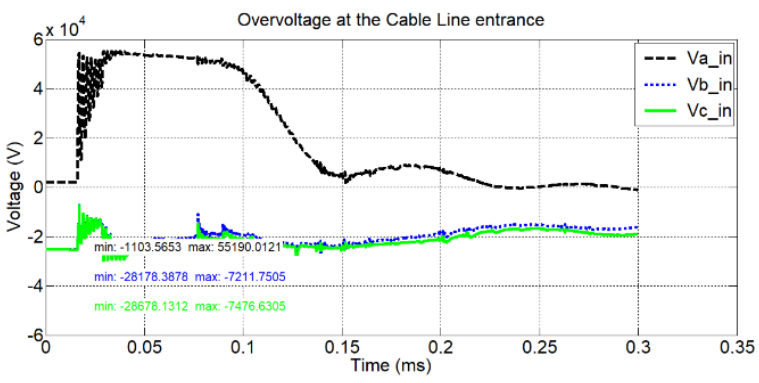

Fig. 23. Overvoltage on cable core after using SPD2

\section{Conclusion}

The paper has studied the overvoltage caused by lightning propagating in wind turbines. Assessment of the influence of atmospheric overvoltage on the insulation of electronic control devices at low voltage, the main insulation of high voltage cables and the insulation of the cable sheath when connected to the overhead line segment (composite structure transmission line).

The effect of using surge protection devices such as SPD1 and SPD2 is considered. The paper describes in detail the simulation model of the system and the voltage transient phenomenon caused by lightning hitting the blades of the wind turbine and mixed-lines using the latest EMTP-RV simulation software.

Future research direction will consider to more accurately model ground electrodes under the effect of lightning current, including time-dependent, timedependent nonlinear ionization in the soil and into the frequency-dependent.

\section{Acknowledgments}

This research is funded by Hanoi University of Science and Technology (HUST) under project number T2017-PC-092.

\section{References}

[1]. Y. Yasuda et al., Surge analysis on wind farm when winter lightning strikes, IEEE Trans. Energy Conversion, Vol. 23, pp 257-262, 2008.

[2]. F. Rachidi et al., A review of current issues in lightning protection of new-generation wind-turbine blades, IEEE Trans. Industrial Electronics, Vol. 55, pp 24892496, 2008 .

[3]. IEC, Wind turbine generation system-24, Lightning Protection,. Tech.Rep.TR61400-24, 2002.

[4]. E. D. Sunde, Earth conduction effects in transmission systems, 2nd ed. New York, NY, USA: DoverPublications, 1968.

[5]. CIGRE WG 33.01, Guide to procedures for estimating the lightning performance of transmission lines, CIGRE technical brochure No. 63, 1991.

[6]. L. V. Bewley, Traveling waves on transmission systems. New York, NY, USA: Dover Publications, 1963.

[7]. A. Soares, M. A. O. Schroeder, and S. Visacro, Transient voltages in transmission lines caused by direct lightning strikes, IEEE Trans. Power Del., vol. 20, no. 2, pp. 1447-1452, Apr. 2005. https://doi.org/10.1109/TPWRD.2004.839214

[8]. CIGRÉ Working Group 33.01, Guide to procedures for estimating the lightning performance of transmission lines, document CIGRÉ Tech. Brochure 63, 1991.

[9]. P. Chowdhuri et al., Parameters of lightning strokes: A review, IEEE Trans. Power Del., vol. 20, no. 1, pp. 346358, Jan. 2005. https://doi.org/10.1109/TPWRD.2004.835039

[10].A. F. Imece et al., Modeling guidelines for fast front transients, IEEE Trans. Power Del., vol. 11, no. 1, pp. 493-506, Jan. 1996. https://doi.org/10.1109/61.484134

[11].J. Takami and S. Okabe, Observational results of lightning current on transmission towers, IEEE Trans. Power Del., vol. 22, no. 1, pp. 547-556, Jan. 2007, http://doi.org/ 10.1109/TPWRD.2006.883006.

[12].IEEE Guide for the Application of Metal-Oxide Surge Arresters for Alternating-Current Systems - Redline, IEEE Std C62.22-2009, vol., no., pp.1-177 July. 2009.

[13].F. M. Gatta, A. Geri, and S. Lauria, Lightning performance improvement of typical $380 \mathrm{kV}$ tower grounding systems, in Proc. International Conference on Grounding and Earthing (GROUND'2006), Maceiò, Brazil, 2006, pp. 321-329.

[14].IEC, Computational guide to insulation coordination and modelling of electrical networks,. Tech. Rep. TR 60071-4, 1st ed., June. 2004. 
[15].A. Geri, F. M. Gatta, S. Lauria, and L. Colla, Lightning performance of long mixed overhead-cable EHVlines,in Proc. 28th International Conference on Lightning Protection (ICLP 2006), Kanazawa, Japan, Sep. 2006

[16].M. Rioual, Short and long air gaps (insulator strings and spark gaps) modelling for lightning studies with EMTP program (EPRI-DCG version 2.0), Research project, final report, Mar.1988.

[17].H. B. Dwight, Calculation of the resistances to ground, Electr. Eng.,vol. 55, pp. 1319-1328, Dec. 1936. https://doi.org/10.1109/EE.1936.6539232

[18].R. Rudenberg, Electrical shock waves in Power Systems. Cambridge,MA: Harvard Univ. Press, 1968. https://doi.org/10.4159/harvard.9780674432390

[19].S. Bourg, B. Sacepe, and T. Debu, Deep earth electrodes in highly resistive ground: Frequency behaviour, in Proc. 1995, IEEE Int. Symp.Electromagn. Compat., pp. 584-589.

[20].L. Grcev and S. Grceva, On HF circuit models of horizontal groundingelectrodes, IEEE Trans. Electromagn. Compat., vol. 51, no. 3, pp. 873-875, Aug. 2009. https://doi.org/10.1109/TEMC.2009.2023330

[21].Basic Impulse Level (BIL) withstand of shield/sheath interrupts, IEEE Standard 575TM-2014.

[22].IEC, Insulation coordination - Part 1: Definitions, principles and rules.IEC Standard 60071-1, 2011.

[23].A. Ametani, Wave Propagation Characteristics of Cables, IEEE Transactions on Power Apparatus andSystems, vol. PAS-99, no. 2, pp. 499-505, Mar. 1980,

https://doi.org/10.1109/TPAS.1980.319685. 